\title{
Impact of variceal eradication on rebleeding and prognosis in cirrhotic patients undergoing secondary prophylaxis
}

\author{
Xing Wang ${ }^{1,2 \#}$, Jinni Luo ${ }^{1,2 \#}$, Chuan Liu ${ }^{3 \#}$, Yanna Liu ${ }^{4}$, Xiaoying Wu ${ }^{1,2}$, Fengping Zheng ${ }^{1,2}$, Zhuofu Wen ${ }^{1,2}$, \\ Hong Tian ${ }^{1,2}$, Xiuqing $\mathrm{Wei}^{1,2}$, Yunwei Guo ${ }^{1,2}$, Jianzhong $\mathrm{Li}^{1,2}$, Xiaoliang Chen ${ }^{1,2}, \mathrm{Jin}_{\mathrm{Tao}}{ }^{1,2}$, Xiaolong $\mathrm{Qi}^{{ }^{4} \wedge}$, \\ Bin $W_{u^{1,2} \wedge}$
}

${ }^{1}$ Department of Gastroenterology, The Third Affiliated Hospital of Sun Yat-sen University, Guangzhou, China; ${ }^{2}$ Guangdong Province Engineering and Technology Research Center of Digestive Endoscopy, Guangzhou, China; ${ }^{3}$ Department of Gastroenterology, Zhujiang Hospital, Southern Medical University, Guangzhou, China; ${ }^{4}$ CHESS Center, Institute of Portal Hypertension, The First Hospital of Lanzhou University, Lanzhou, China

Contributions: (I) Conception and design: X Wang, X Qi, B Wu; (II) Administrative support: X Qi, B Wu; (III) Provision of study materials or patients: X Wu, F Zheng, Z Wen, H Tian, X Wei, Y Guo, J Li, X Chen, J Tao, B Wu; (IV) Collection and assembly of data: X Wang, J Luo, C Liu; (V) Data analysis and interpretation: X Wang, J Luo, C Liu, Y Liu, X Qi, B Wu; (VI) Manuscript writing: All authors; (VII) Final approval of manuscript: All authors.

\#These authors contributed equally to this work.

Correspondence to: Bin Wu, MD, PhD. Professor and Chairman, Department of Gastroenterology, The Third Affiliated Hospital of Sun Yat-sen University, 600 Tianhe Road, Guangzhou 510630, China. Email: wubin6@mail.sysu.edu.cn; Xiaolong Qi, MD. Professor of Medicine, Chair of CHESS (Chinese Portal Hypertension Diagnosis and Monitoring Study Group), CHESS Center, Institute of Portal Hypertension, The First Hospital of Lanzhou University, Lanzhou 730000, China. Email: qixiaolong@vip.163.com.

Background: Endoscopic therapy has been widely applied to prevent variceal rebleeding, but data addressing the effect of endoscopic variceal eradication (VE) are lacking. We aimed to clarify the clinical impact of $\mathrm{VE}$ and reveal the long-term incidence and mortality of gastrointestinal rebleeding.

Methods: This prospective study included 228 cirrhotic patients who underwent secondary prophylaxis for variceal bleeding and achieved VE through a systematic procedure we proposed as endoscopic sequential therapy (EST). Rebleeding rates before and after VE were compared and cumulative incidence of rebleeding and mortality were calculated using the Kaplan-Meier method. A logistic regression model and P for trend were used to investigate the optimal time limit for $\mathrm{VE}$.

Results: During a median (interquartile range) follow-up duration of 33.0 (23.0-48.75) months, rebleeding was identified in 28 patients (12.3\%) after VE and in 27 patients (11.8\%) during endoscopic sessions. The cumulative incidence of rebleeding before and after $\mathrm{VE}$ was $8.4 \%$ and $1.8 \%$ at 6 months, and $14.9 \%$ and $4.0 \%$ at 1 year respectively $(\mathrm{P}<0.001)$. The long-term incidence of all-cause/variceal rebleeding following $\mathrm{VE}$ was $10.4 \% / 9.1 \%$, and $31.5 \% / 23.5 \%$ at 2 and 5 years respectively. Eleven patients $(4.8 \%)$ died and the 5 -year mortality was $9.3 \%$. VE achieved within 6 months was associated with fewer rebleeding events compared to $\mathrm{VE}$ achieved after 6 months $(5.5 \%$ vs. $20.0 \%, \mathrm{P}=0.002)$, while logistic regression revealed an overall increasing trend in the odds ratio of rebleeding ( $v s$. patients with $\mathrm{VE}$ time $\leq 6$ months) for patients with $6<$ $\mathrm{VE}$ time $\leq 12$ months and VE time $>12$ months ( $\mathrm{P}$ for trend $<0.001$ ).

Conclusions: VE further reduces rebleeding based on routine endoscopic prophylaxis and improves longterm prognosis. VE within 6 months seems to be the optimal timing and should therefore be advocated.

Keywords: Variceal hemorrhage; variceal eradication (VE); endoscopy; sequential therapy; liver cirrhosis

\footnotetext{
^ ORCID: Xing Wang, 0000-0002-5950-4591; Xiaolong Qi, 0000-0002-3559-5855; Bin Wu, 0000-0001-9039-9681.
} 
Submitted Apr 20, 2020. Accepted for publication Dec 31, 2020.

doi: 10.21037/atm-20-3401

View this article at: http://dx.doi.org/10.21037/atm-20-3401

\section{Introduction}

Esophagogastric variceal bleeding (EGVB) is a serious decompensating event in liver cirrhosis with poor longterm prognosis, accounting for $20 \%$ of 5 -year mortality as an isolated complication and over $80 \%$ of 5 -year mortality when associated with other complications (1). If untreated with proper subsequent secondary prophylaxis after hemostasis, recurrent EGVB occurs in $60 \%$ of patients, mostly within 1-2 years following the first episode of bleeding (2). In past decades, endoscopic therapies, including endoscopic band ligation (EBL), endoscopic cyanoacrylate injection (ECI) and endoscopic injection sclerotherapy (EIS), have been developed and widely applied as first-line treatment options for EGVB, leading to a substantial reduction in rebleeding and subsequent deaths $(1,3,4)$. However, despite the therapeutic improvements, long-term rebleeding rates are still unsatisfactory and are reportedly as high as $30 \%$ at 1 year and $50 \%$ at 5 years $(5-7)$.

Variceal eradication (VE), manifested as invisible or small residual esophagogastric varices (EGV), has been recommended as the therapeutic goal of endoscopic treatment and can be achieved by repeated sessions of operational endoscopies $(1,3)$. Limited data show that VE reduces the rebleeding rate by more than $50 \%$ compared to the absence of endoscopic prophylaxis $(8,9)$. However, the actual impact of VE on prognosis has seldom been evaluated in cohorts of patients receiving endoscopic prophylaxis. Moreover, the additional long-term benefit following $\mathrm{VE}$ achievement has not been studied. Meanwhile, a standard endoscopic protocol integrating treatment for both esophageal varices $(\mathrm{EV})$ and gastric varices $(\mathrm{GV})$ has not been established $(5,10)$. Therefore, our study aimed to clarify the impact of endoscopic VE on rebleeding and mortality in cirrhotic patients undergoing secondary prophylaxis and proposes a systematic procedure, endoscopic sequential therapy (EST), highlighting complete eradication of EV and GV. We present the following article in accordance with the STROBE reporting checklist (available at http://dx.doi.org/10.21037/atm-20-3401).

\section{Methods}

\section{Study design and participants}

This prospective cohort study was conducted at a university-affiliated referral hospital. Consecutive patients with liver cirrhosis who underwent EBL, ECI or EIS as endoscopic treatment for EGV between October 2011 and August 2018 were screened for the study. Patients undergoing the first session of operational endoscopy for secondary prophylaxis of variceal bleeding in our endoscopy center were included. Patients were excluded if they met any of the following criteria: absence of variceal bleeding history; endoscopic treatment during the acute bleeding phase; history of prior hepatectomy, splenectomy, radiointervention therapy or liver transplantation; coexistence of advanced hepatocellular carcinoma (HCC); sole presence of isolated $\mathrm{GV}$; or refusal to participate in the study. The patient selection flow chart is shown in Figure 1. This study was approved by the institutional review board of The Third Affiliated Hospital of Sun Yat-sen University (No. 2-79) and conducted in accordance with the Declaration of Helsinki (as revised in 2013). Written informed consent was obtained from all study participants.

\section{Data collection and study follow-up}

Baseline demographic, clinical and laboratory characteristics, along with endoscopic features, were collected. Endoscopic features were assessed based on the general rules of recording endoscopic findings for EGV (11). After baseline assessment, all recruited patients underwent an initial endoscopic treatment session for EGV, and the sessions were repeated every 4-6 weeks thereafter until eradication of the varices. EV eradication was defined as the absence of visible continuous varices upon air insufflation or any residual varices with red signs that could be ligated or injected. GV eradication was defined as the time when the entire GV became solidified by cyanoacrylate injection or as the absence of any size of varices with red signs $(11,12)$. All patients included in the final analysis successfully achieved 


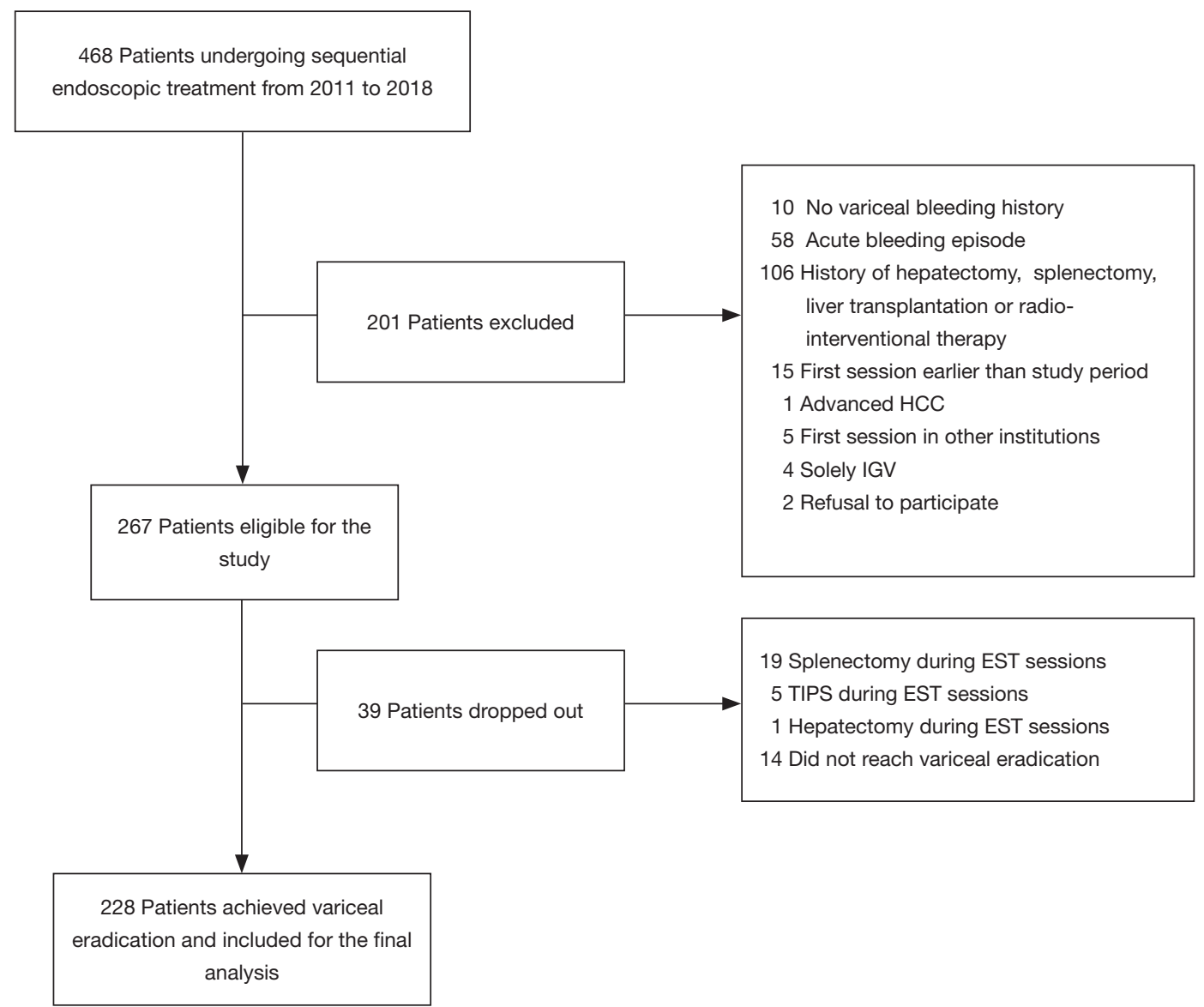

Figure 1 Study population selection. A total of 228 patients were finally included out of 468 patients undergoing endoscopic treatment for esophagogastric varices. HCC, hepatocellular carcinoma; EST, endoscopic sequential therapy; IGV, isolated gastric varices; TIPS, transjugular intrahepatic portosystemic shunt.

VE, which had to be agreed upon by two experienced endoscopists. Patients were followed up every 3-6 months with clinical assessment including endoscopic examinations, laboratory tests, concomitant non-endoscopic treatments, and development of gastrointestinal bleeding or death.

\section{Study outcomes}

The primary outcome was recurrent upper gastrointestinal bleeding or death at 1, 2 or 5 years after VE achievement, while variceal rebleeding was considered a subtype of allcause gastrointestinal rebleeding. The secondary outcome was 6-month and 1-year rebleeding during endoscopic sessions before VE achievement. Outcome data were obtained primarily from study follow-up records. Patients who did not visit the clinic regularly were followed up through telephone inquiry. Rebleeding was defined as overt gastrointestinal bleeding (e.g., hematemesis, melena) associated with unstable vital signs (systolic blood pressure $\leq 90 \mathrm{mmHg}$ or pulse $\geq 110 \mathrm{bpm}$ ), a significant drop in hemoglobin (at least $20 \mathrm{~g} / \mathrm{L}$ ), the need for blood transfusion with hemoglobin less than $70 \mathrm{~g} / \mathrm{L}$, and/or endoscopic verified bleeding (5). In addition, cases achieving VE within 6 months were categorized into the fast-VE group, while cases achieving $\mathrm{VE}$ after more than 6 months were categorized into the slow-VE group.

\section{Performance of EST}

EST is a combination strategy developed in our department defined as ECI (when necessary) sequenced by repeated sessions of EBL and EIS (when necessary). In general, 
$\mathrm{GV} \geq 10 \mathrm{~mm}$ in diameter and/or with high-risk bleeding stigmata were treated with ECI at the first visit, followed by repeated EBL for EV or proper GV during the same session or in subsequent sessions every 4-6 weeks. When $\mathrm{EV}$ became discontinuous or were surrounded by scars, under which condition the EBL was inapplicable, EIS was performed for the remnant $\mathrm{EV}$ to achieve VE. ECI was performed using a GIF-H260 (Olympus Corp., Tokyo, Japan) or EG-L590 (Fujinon Inc., Saitama, Japan) endoscope and a 23-gauge disposable injection needle catheter (1,800 $\mathrm{mm}$ in length). A mixture of N-butyl-2cyanoacrylate (Compont Medical Corp., Beijing, China) and lipiodol (Guerbet, Villepinte, France) at a 1:1 ratio was injected with the total dosage decided by the operator depending on the type and size of the GV. EBL was performed using multiband devices (Wilson-Cook Medical Inc., Winston-Salem, NC, or SpeedBand, Boston Scientific Corp., Natick, MA). The procedure started at or just below the gastroesophageal junction and each varix was ligated, but no more than 7 bands were applied per session. EIS was performed using $1 \%$ lauromacrogol injection (Tianyu Chang'an Corp., Xi'an, China). No more than $5 \mathrm{~mL}$ sclerosant was injected into each site, and the total amount did not exceed $20 \mathrm{~mL}$ per session. The schematic workflow and endoscopic views of EST are shown in Figures 2 and 3, respectively. The performance principle was in accordance with European and Chinese guidelines while considering clinical experiences and local expertise (3,4,9,13-16). All procedures were performed by the same medical team, and the specific option for each endoscopic session was selected at the endoscopist's discretion.

\section{Statistical analysis}

Categorical variables were compared using Chi-square or Fisher's exact test when appropriate, and quantitative variables were compared using Student's $t$-test or MannWhitney U test, as applicable. The Kaplan-Meier method was used to estimate the cumulative incidence of rebleeding before and after VE. To illustrate the impact of VE on rebleeding, the log-rank test was used to compare the incidence of rebleeding before and after $\mathrm{VE}$, as shown in different survival curves. Meanwhile, variceal rebleeding was considered a subtype of all-cause rebleeding, and the log-rank test was also performed to compare the incidence of variceal rebleeding before and after VE. In the rebleeding analysis, the endpoint was rebleeding, and data were censored at the time of last clinical visit, death or transfer to other non-endoscopic therapies, except locoregional therapies for small HCC. In the mortality analysis, the endpoint was death or liver transplantation, and data were censored at the time of last clinical visit or when transferred to other therapies as above. Moreover, rebleeding and mortality rates were compared between the fast-VE and slow-VE groups using the Chi-square test, and logistic regression was used to investigate the optimal timing for VE. A two-tailed $\mathrm{P}$ value less than 0.05 was considered statistically significant. All data were analysed using SPSS version 21.0 software (IBM Corp., Armonk, NY) and R statistics version 3.4.1 (R Core Team, Vienna, Austria).

\section{Results}

\section{Patient characteristics and endoscopic treatment strategies}

During the study period, 468 consecutive patients underwent sequential endoscopic treatment. Among them, 201 patients were excluded based on the exclusion criteria, and another 39 patients were excluded during the EST sessions. As a result, 228 patients $(85.4 \%)$ achieved VE and were included in the study cohort (Figure 1) with a median (interquartile range, IQR) follow-up time of 33.0 (23.048.75) months. The cohort consisted of 178 (78.1\%) men and $50(21.9 \%)$ women with a mean age of $49.3 \pm 10.5$ years. One hundred thirty-three (58.3\%) patients had Child-Pugh A cirrhosis, 95 (41.7\%) patients had Child-Pugh B or C cirrhosis, and 9 (3.9\%) patients had Model for End-Stage Liver Disease (MELD) scores of 20 or above. Hepatitis $\mathrm{B}$ infection was the major underlying etiology (62.7\%), followed by alcohol consumption (11.8\%). Eighty-five $(37.3 \%)$ patients were receiving non-selective beta-blockers (NSBBs) on admission. Two patients were diagnosed with small HCC without radiographic evidence of macrovascular invasion, and 14 patients had portal vein thrombosis at enrollment. Clinical and endoscopic characteristics of the included patients are shown in Table 1. On average, 3.6 sessions of EST were performed to achieve VE for each patient, and the mean time duration required was 8.5 months. EBL was the mainstay treatment option and was performed in nearly all patients $(97.8 \%)$. The total number of bands applied per patient was 18 (Table S1).

\section{Clinical outcome of rebleeding over time}

A total of 28 patients (12.3\%) experienced rebleeding after VE. Among the rebleeding episodes, 17 (60.7\%) 


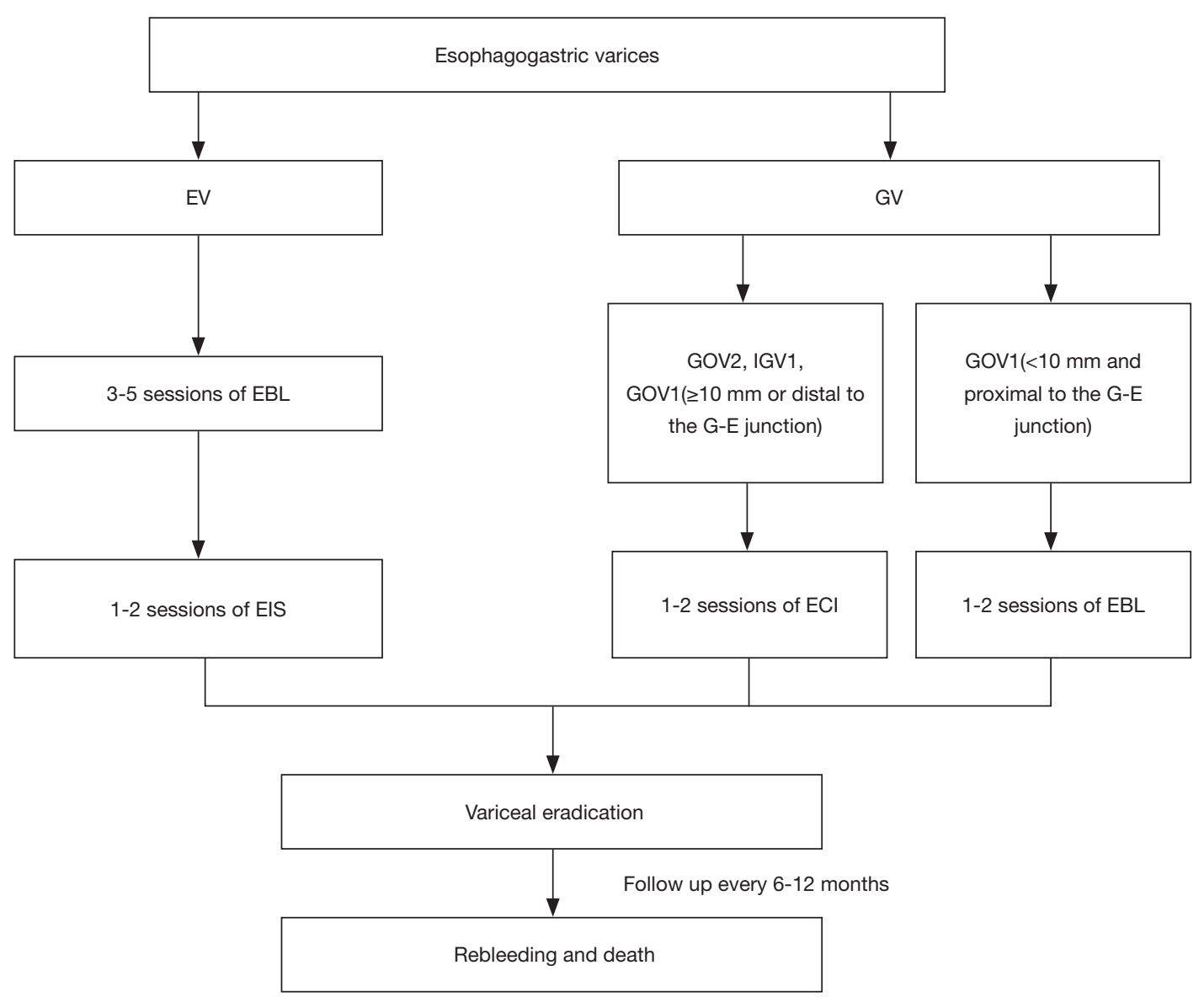

Figure 2 Workflow of endoscopic sequential therapy and subsequent follow up. EV, esophageal varices; GV, gastric varices; EBL, endoscopic band ligation; EIS, endoscopic injection sclerotherapy; ECI, endoscopic cyanoacrylate injection; GOV, gastroesophageal varices; $\mathrm{IGV}$, isolated gastric varices.

involved bleeding from varices (7 EV, $7 \mathrm{GV}, 3 \mathrm{EV}$ and GV), and 11 involved other causes (Table 2). The median (IQR) time duration from eradication to rebleeding was 16.5 (9.25-27.5) months. The cumulative incidence of rebleeding at 1, 2 and 5 years was $4.0 \%, 10.4 \%$, and $31.5 \%$, respectively. Regarding variceal rebleeding specifically, the incidence at the above corresponding years was $2.6 \%, 9.1 \%$, and $23.5 \%$, respectively (Figure 4 ). In contrast, 27 patients $(11.8 \%)$ presented with rebleeding during the endoscopic sessions before $\mathrm{VE}$, and most of the bleeding cases were variceal-related (23 cases, $85.2 \%)$. The majority of patients achieved VE within 12 months (182 cases, $79.8 \%$ ). The cumulative incidence of rebleeding before $\mathrm{VE}$ at 6 months and 1 year was $8.4 \%$ and $14.9 \%$, respectively, which was significantly higher than rebleeding after VE $(\log -$ rank $\mathrm{P}<0.001)$ (Figure $5 A$ ). When considering variceal rebleeding cases alone, short- term rebleeding before $\mathrm{VE}$ was also higher than after $\mathrm{VE}$ (log-rank $\mathrm{P}<0.001)$ (Figure 5B). With respect to treatment selection, patients receiving endoscopy plus NSBBs combination therapy showed similar rebleeding rates compared to endoscopic therapy alone, whether measured as all-cause rebleeding or variceal rebleeding during the follow-up period after VE (log-rank $\mathrm{P}=0.05$ and 0.06 , respectively) (Figures S1,S2).

\section{Clinical outcome of death over time and patients transferred to non-endoscopic therapies}

Eleven patients $(4.8 \%)$ died during the entire followup period. Causes of death included HCC (4 cases), liver failure (3 cases), systemic organ failure (2 cases) and cerebral hemorrhage (1 case). Only one case died of variceal bleeding (Table 2). Accordingly, the cumulative incidence 


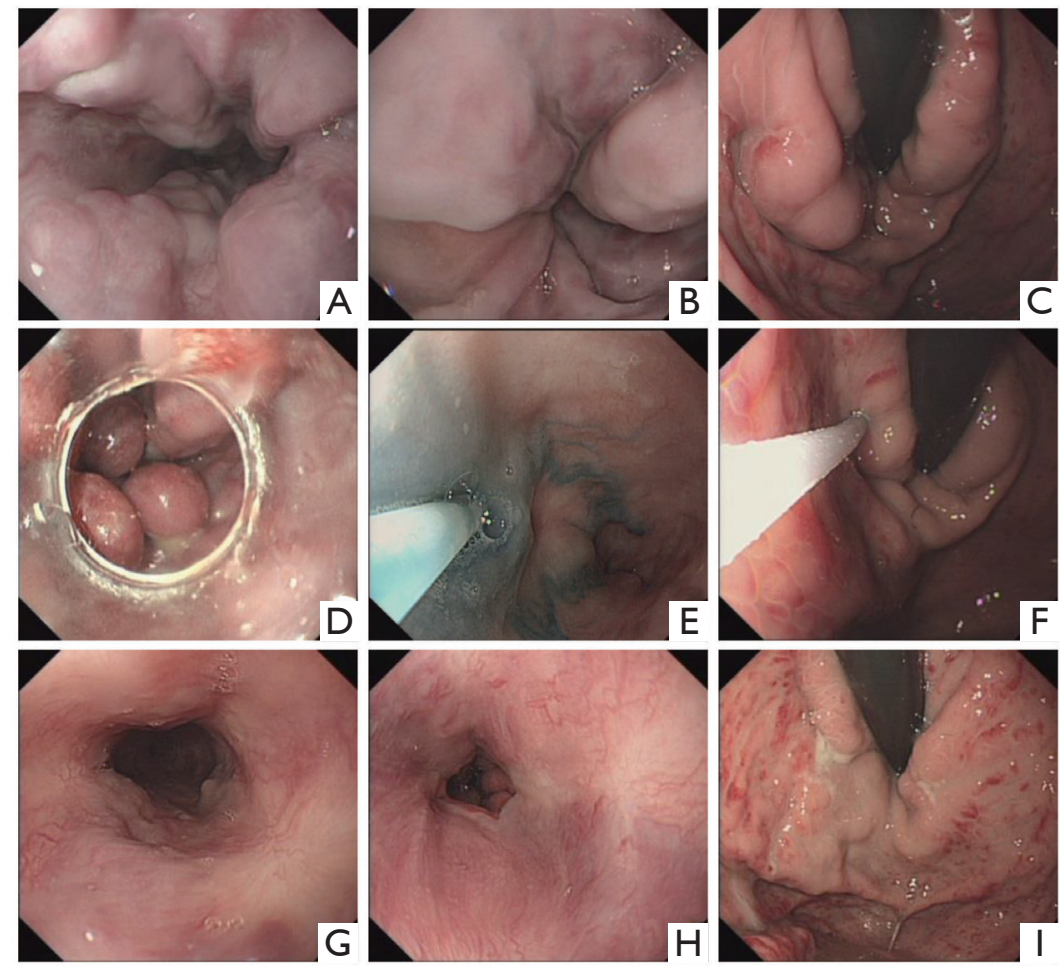

Figure 3 Endoscopic views in different phases of endoscopic sequential therapy. (A,B) Severe EV in the middle and lower section of esophagus before EBL; (C) severe GV in the stomach along the lesser curvature before ECI; (D) performance of EBL; (E) performance of EIS on remnant EV with tracer of methylene blue solution after repeated EBL; (F) performance of ECI; (G,H) complete eradication of $\mathrm{EV}$ in the middle and lower section of esophagus after two sessions of EBL and one session of EIS; (I) complete eradication/consolidation of GV after one session of ECI. EV, esophageal varices; GV, gastric varices; EBL, endoscopic band ligation; EIS, endoscopic injection sclerotherapy; ECI, endoscopic cyanoacrylate injection.

of all-cause mortality at 1, 2 and 5 years was $0.9 \%, 4.4 \%$ and $9.3 \%$, respectively. No deaths occurred during EST sessions before VE. In contrast, 24 patients (10.5\%) were transferred to surgeries, interventional portosystemic shunt therapies and liver transplantations. Meanwhile, on-site endoscopy failure, which was defined as bleeding within 5 days of initial endoscopy, did not occur in our study. During the entire follow-up, no serious adverse events in the forms of perforation, strictures, chest empyema or pericardial effusion were observed, whereas transient dysphagia, chest pain and epigastric pain were occasional but did not require specific treatment.

\section{Comparison of rebleeding and mortality for the fast-VE group and slow-VE group}

One hundred twenty-eight patients (56.1\%) achieved VE within 6 months, categorized as the fast-VE group, while the remaining 100 patients were categorized as the slow-VE group. Seven cases (5.5\%) in the fast-VE group and 20 cases $(20 \%)$ in the slow-VE group experienced rebleeding events during endoscopic sessions (Chi-square $\mathrm{P}=0.002$ ) (Figure 6). Logistic regression showed that prolonged eradication time significantly increased the risk of rebleeding compared to restricted VE time within 6 months (OR 2.88, 95\% CI: $0.95-$ 8.70 for the group of $6<\mathrm{VE}$ time $\leq 12$ months; OR 5.91, $95 \%$ CI: $2.20-15.89$ for the group of VE time $>12$ months) with $\mathrm{P}$ for trend $<0.001$ (Table 3). However, regarding rebleeding and mortality after VE, the two groups showed no significant differences (Chi-square $\mathrm{P}=0.603$ for rebleeding; Chisquare $\mathrm{P}=0.660$ for mortality). Furthermore, no significant differences in baseline clinical parameters were observed between the two groups (Table S2).

\section{Discussion}

This study clarified the impact of $\mathrm{VE}$ on reducing recurrent bleeding in an endoscopic prophylaxis cohort. The natural 
Table 1 Baseline characteristics of patients on secondary prophylaxis

\begin{tabular}{|c|c|}
\hline Characteristic & Total cohort $(n=228)$ \\
\hline Age (years) & $49.3 \pm 10.5$ \\
\hline Sex (male, \%) & $178(78.1)$ \\
\hline \multicolumn{2}{|l|}{ Etiology of liver cirrhosis, n (\%) } \\
\hline HBV & $143(62.7)$ \\
\hline HCV & $14(6.1)$ \\
\hline Alcohol & $27(11.8)$ \\
\hline Alcohol + viral & $18(7.9)$ \\
\hline Others & $26(11.4)$ \\
\hline Hypertension, n (\%) & $16(7.0)$ \\
\hline Diabetes mellitus, n (\%) & $37(16.2)$ \\
\hline Ascites, n (\%) & $95(41.7)$ \\
\hline Hepatocellular carcinoma, n (\%) & $2(0.9)$ \\
\hline Portal vein thrombosis, n (\%) & $14(6.1)$ \\
\hline Use of $\beta$-blocker, $\mathrm{n}(\%)$ & 85 (37.3) \\
\hline \multicolumn{2}{|l|}{ Laboratory test } \\
\hline Hemoglobin level (g/L) & $90.1 \pm 22.2$ \\
\hline Platelet count $\left(\times 10^{9} / L\right)$ & $87.0 \pm 43.3$ \\
\hline ALT level (U/L) & $39.5 \pm 42.7$ \\
\hline Total bilirubin level ( $\mu \mathrm{mol} / \mathrm{L})$ & $26.2 \pm 27.3$ \\
\hline Prothrombin INR & $1.37 \pm 0.4$ \\
\hline \multicolumn{2}{|l|}{ Child-Pugh classification, n (\%) } \\
\hline$A$ & $133(58.3)$ \\
\hline B & $79(34.6)$ \\
\hline C & $16(7.0)$ \\
\hline \multicolumn{2}{|l|}{ MELD score, n (\%) } \\
\hline $0-9$ & $84(36.8)$ \\
\hline $10-19$ & $135(59.2)$ \\
\hline $20-30$ & $9(3.9)$ \\
\hline \multicolumn{2}{|c|}{ Endoscopic findings at initial therapy, n (\%) } \\
\hline Type, EV + GV/EV & $185 / 43(81.1 / 18.9)$ \\
\hline Location, $\mathrm{Li}+\mathrm{Lm}$ or $\mathrm{Li} / \mathrm{Li}+\mathrm{Lm}+\mathrm{Ls}$ & 214/14 (93.9/6.1) \\
\hline EV size, $F 1+F 2 / F 3$ & $17 / 211(7.5 / 92.5)$ \\
\hline GV size, F1 + F2/F3 & 88/99 (38.6/43.4) \\
\hline EV, red sign, n (\%) & $214(93.9)$ \\
\hline GV, red sign, n (\%) & $110(48.2)$ \\
\hline
\end{tabular}

Data presented as mean \pm SD or number of patients (percentage) where appropriate. MELD, Model for End-Stage Liver Disease; INR, international normalized ratio; EV, esophageal varices; GV, gastric varices. history of cirrhotic patients in terms of rebleeding and mortality following VE was also delineated. To the best of our knowledge, real world prognostic data with clearly defined endoscopic eradication of both $\mathrm{EV}$ and GV have never been reported. Moreover, an eradication time limit of 6 months was found to be optimal, resulting in less interval rebleeding during endoscopic sessions.

According to prior studies, endoscopic therapy for secondary prophylaxis is still associated with a $20 \%$ to $30 \%$ rate of 1-year rebleeding, and procedure-related deaths might even occur $(5-7,17,18)$. Therefore, the therapeutic goal of VE was proposed adhering to clinical guidelines. Recent data showed that the rebleeding proportion before $\mathrm{VE}$ was $24 \%$ to $32 \%(8,19)$, which was much higher than in our study $(12 \%)$. In contrast, the rebleeding proportion after $\mathrm{VE}$ was reported to be $11.3 \%$ to $25 \%(8,20)$ and our data were within this range (12.3\%). However, these studies only treated $\mathrm{EV}$ and did not provide rebleeding probability in a time-dependent manner, making the impact of a complete VE still unclear. Although the overall percentages before and after eradication were similar in our study, the beneficial impact became marked when taking the follow-up period into account, and an additional $10 \%$ decrease in the one-year rebleeding incidence was observed. On the other hand, most previous studies analysed data on EV alone, while few studies evaluated patients undergoing endoscopic treatment for both $\mathrm{EV}$ and coexisting junctional or fundal GV $(5,10)$. Considering that GV occurs in $50 \%$ of cirrhotic patients and contributes to $10-20 \%$ of variceal bleeding $(1,4,21,22)$, a complete occlusion of both EV and GV achieved by EST in our study was a sound and more ideal endoscopic endpoint than EV eradication alone. Since the mortality is still as high as $20 \%$ at 6 weeks after a separate bleeding episode, preventing bleeding during endoscopic intervals is of great importance and aggressive $\mathrm{VE}$ in our study should be considered favorable for waning rebleeding and mortality $(23,24)$.

There was no standard time limit or number of sessions needed for VE, but most studies have reported a mean number of 3-6 sessions, and the recommended interval for band ligation is $2-4$ weeks $(1,3,4,8,19,25)$. In our study, we believe that 6 months is an optimal VE time limit because a large group of our patients underwent concurrent cyanoacrylate injection for $\mathrm{GV}$, which may require 6-8 weeks for better reassessment on endoscopy. When taking time into account, the rebleeding rate before $\mathrm{VE}$ is still unsatisfactory, and we found that most bleeding events occurred in the slow-VE group. This might be due to 


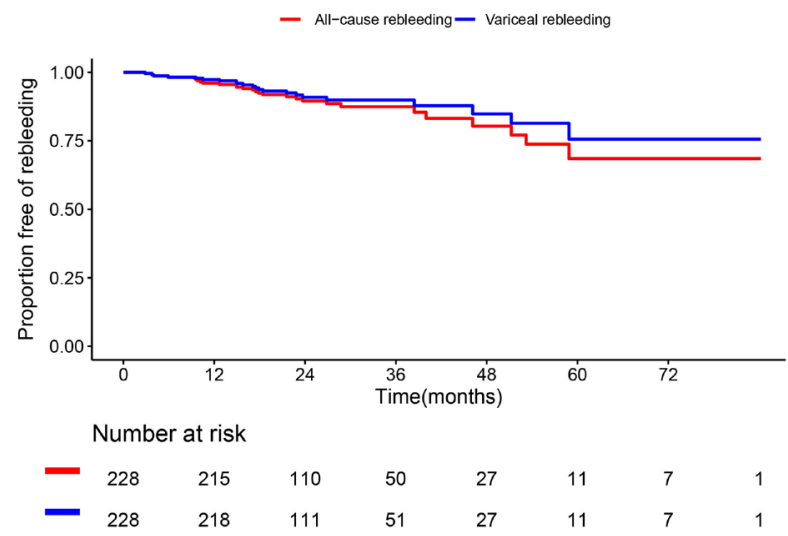

Figure 4 Kaplan-Meier analysis of rebleeding after variceal eradication. Survival curves of patients showing cumulative incidence of allcause rebleeding (red) and variceal rebleeding (blue).

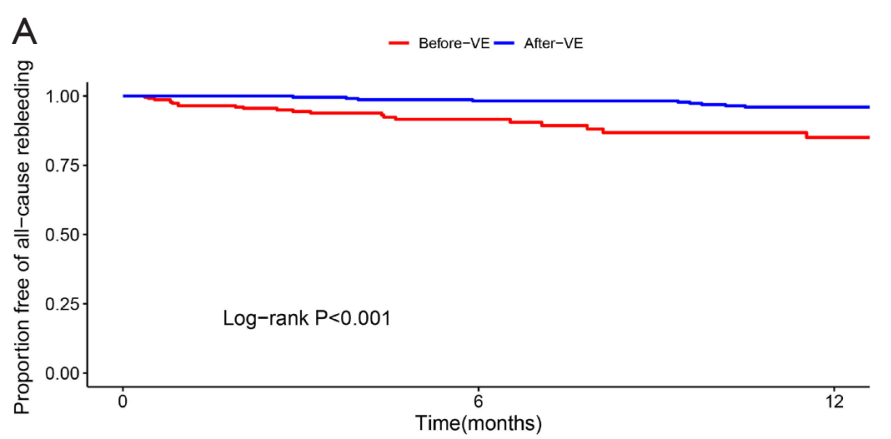

Number at risk

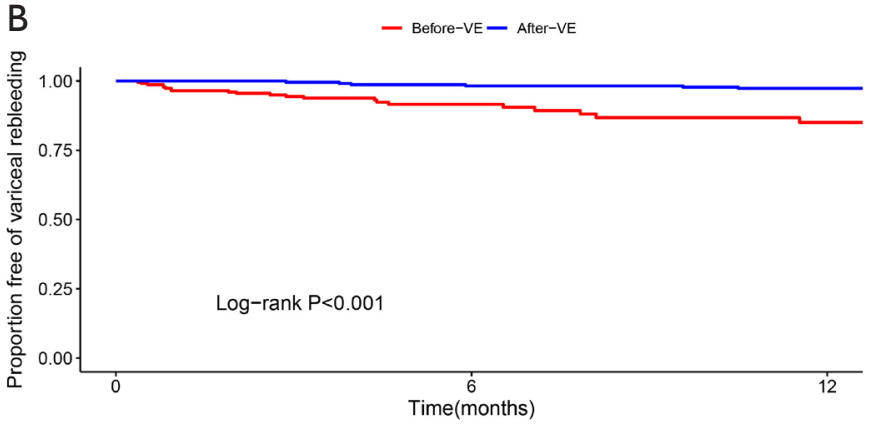

Number at risk

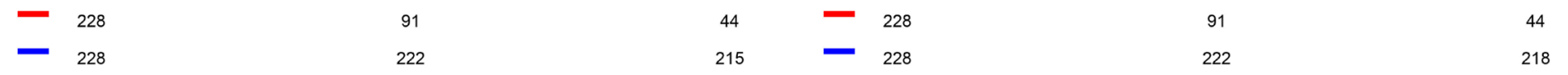

Figure 5 Kaplan-Meier analysis of rebleeding before and after variceal eradication. (A) Survival curves of patients showed that the cumulative incidences of all-cause rebleeding before VE at 6 months and 1 year were significantly higher than those of rebleeding after VE. (B) When considering variceal rebleeding cases alone, rebleeding incidences before $\mathrm{VE}$ at 6 months and 1 year were also significantly higher than those of rebleeding after VE. VE, variceal eradication.

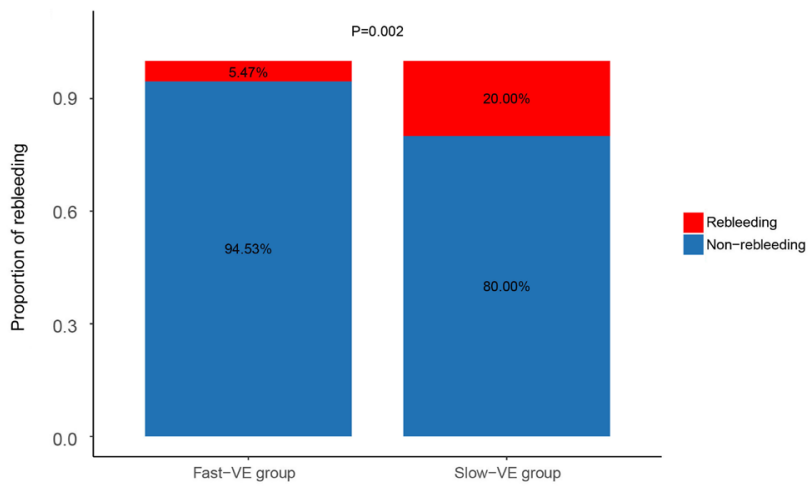

Figure 6 Comparison of rebleeding risk between fast-VE group and slow-VE group. Fast-VE group (VE achieved within 6 months) presented with less rebleeding events than slow-VE group (VE achieved after more than 6 months). VE, variceal eradication. 
Table 2 Clinical outcomes of patients on secondary prophylaxis

\begin{tabular}{|c|c|}
\hline Outcomes & Total cohort $(n=228)$ \\
\hline $\begin{array}{l}\text { Overall follow-up duration, (months, } \\
\text { median, IQR) }\end{array}$ & $33.0(23.0-48.75)$ \\
\hline $\begin{array}{l}\text { Follow-up duration after eradication, } \\
\text { (months, median, IQR) }\end{array}$ & $24.0(17.0-35.0)$ \\
\hline $\begin{array}{l}\text { Duration to rebleeding }{ }^{1} \text { (months, median, } \\
\text { IQR) }\end{array}$ & $16.5(9.25-27.5)$ \\
\hline Rebleeding source, n (\%) & $28(12.3)$ \\
\hline EV & $7(3.1)$ \\
\hline GV & $7(3.1)$ \\
\hline $\mathrm{EV}+\mathrm{GV}$ & $3(1.3)$ \\
\hline Procedure-related & $1(0.4)$ \\
\hline Peptic ulcer & $3(1.3)$ \\
\hline Others & $3(1.3)$ \\
\hline Unknown & $4(1.8)$ \\
\hline \multicolumn{2}{|l|}{ Rebleeding treatment, n (\%) } \\
\hline Medical & $13(5.7)$ \\
\hline Endoscopic & $14(6.1)$ \\
\hline Surgical or radio-interventional & $1(0.4)$ \\
\hline $\begin{array}{l}\text { Duration to interval rebleeding }{ }^{2} \text { (months, } \\
\text { median, IQR) }\end{array}$ & $4(0-11)$ \\
\hline Interval rebleeding source, n (\%) & $27(11.8)$ \\
\hline EV & $10(4.4)$ \\
\hline GV & $9(3.9)$ \\
\hline $\mathrm{EV}+\mathrm{GV}$ & $4(1.8)$ \\
\hline Procedure-related & $2(0.7)$ \\
\hline Unknown & $2(0.7)$ \\
\hline Transfer to other therapies, $\mathrm{n}(\%)$ & $24(10.5)$ \\
\hline Surgical therapy & $20(8.8)$ \\
\hline Radio-interventional therapy & $3(1.3)$ \\
\hline Liver transplantation & $1(0.4)$ \\
\hline Duration to death ${ }^{3}$ (months, median, IQR) & $21.0(12.0-29.0)$ \\
\hline Cause of death, $\mathrm{n}(\%)$ & $11(4.8)$ \\
\hline Variceal bleeding & $1(0.4)$ \\
\hline Non-variceal bleeding & $0(0)$ \\
\hline Hepatocellular carcinoma & $4(1.8)$ \\
\hline Liver failure & $3(1.3)$ \\
\hline Others & $3(1.3)$ \\
\hline
\end{tabular}

${ }^{1}$, for rebleeding cases after variceal eradication; ${ }^{2}$, for rebleeding cases during endoscopic sessions; ${ }^{3}$, for dead cases. Data presented as median and IQR or number of patients (percentage) where appropriate. IQR, interquartile range; EV, esophageal varices; GV, gastric varices; EBL, Endoscopic banding ligation; $\mathrm{ECl}$, endoscopic cyanoacrylate injection; EIS, endoscopic injection sclerotherapy.
Table 3 Rebleeding risk stratified by different time periods used for variceal eradication

\begin{tabular}{ll}
\hline Time for VE & Rebleeding risk before VE, OR $(95 \% \mathrm{Cl})$ \\
\hline$\leq 6$ months & 1 \\
$6<$ time $\leq 12$ months & $2.88(0.95-8.70)$ \\
$>12$ months & $5.91(2.20-15.89)$ \\
$P$ for trend & $<0.001$ \\
\hline
\end{tabular}

VE, variceal eradication; OR, odds ratio; Cl, confidence interval.

poor compliance of this group of patients, having a greatly prolonged endoscopic interval and extended time duration to the eradication, which increased the bleeding risk of remaining high-risk varices awaiting treatment (26-28). Another reason for the high proportion of variceal-related rebleeding before $\mathrm{VE}$ was presumed to be the observed low prevalence of the use of non-selective $\beta$-blockers with less than $40 \%$ in our cohort, while the status of hemodynamic response and long-term drug adherence of these patients were both unknown. In practice, compromised patient compliance is a major threat to clinicians but is very common in chronic liver disease (29-32). However, the improved post-eradication prognosis revealed by our data reflects the advantage of $\mathrm{VE}$, which may even benefit poor compliance patients who do not regularly follow up. In addition, during the endoscopic intervals before VE, we noted that rebleeding events were usually not fatal and could achieve hemostasis with timely endoscopic interventions. Nevertheless, our data strengthened the concept of fast eradication complying with clinical guidelines, and reinforced health education and optimized follow-up management are highly needed.

There are several limitations of our study. First, as a single-center study performed in an academic referral hospital, patient selection bias is inevitable, which may weaken the generalizability of our data. Second, all patients underwent the same endoscopic management strategy and there was no traditional mono EBL therapy group or mono medical treatment group for comparison. Therefore, we failed to compare the efficacy of EST to EBL or medication directly. However, in our study group of over $80 \%$ patients with concomitant high-risk EV and GV, monotherapy was of ethical concern and may hardly be applied in practice. Third, eradication time was longer than previously reported due to poor compliance of some patients, which may impair the efficacy of endoscopy therapy and increase 
interval bleeding events. Therefore, improved patient education is imperative. Fourth, although we screened all cases undergoing EST within the study period, the sample size was still insufficient and may attenuate the statistical power. Lastly, since the included cases were mostly mild to moderate liver disease patients, our findings may not be applicable to populations with more severe liver disease.

\section{Conclusions}

VE further reduces rebleeding based on routine endoscopic prophylaxis and improves long-term prognosis. Rapid VE within 6 months seems to be an optimal time duration for the entire endoscopy period and should therefore be advocated. EST is an effective way to achieve complete VE in real-world practice. Multicenter studies comprising larger population sizes remain highly warranted to further validate the beneficial effect of VE and the efficacy of EST compared to other strategies in the future.

\section{Acknowledgments}

The authors would like to thank every physician in our department for the helpful discussions and AJE for its linguistic assistance during the preparation of this manuscript.

Funding: This study was supported by the National Natural Science Foundation of China (U1501224), the Natural Science Foundation of Guangdong Province Team Project (2018B030312009), the Science and Technology Developmental Foundation of Guangdong Province (2017B020226003), and the Science and Technology Program of Guangzhou City (201604020118). The funders had no role in the design, implementation or interpretation of results of this study.

\section{Footnote}

Reporting Checklist: The authors have completed the STROBE reporting checklist. Available at http://dx.doi. org/10.21037/atm-20-3401

Data Sharing Statement: Available at http://dx.doi. org/10.21037/atm-20-3401

Conflicts of Interest: All authors have completed the ICMJE uniform disclosure form (available at http://dx.doi. org/10.21037/atm-20-3401). The authors have no conflicts of interest to declare.

Ethical Statement: The authors are accountable for all aspects of the work in ensuring that questions related to the accuracy of integrity of any part of the work are appropriately investigated and resolved. This study was approved by the institutional review board of The Third Affiliated Hospital of Sun Yat-sen University (No. 2-79) and conducted in accordance with the Declaration of Helsinki (as revised in 2013). Written informed consent was obtained from all study participants.

Open Access Statement: This is an Open Access article distributed in accordance with the Creative Commons Attribution-NonCommercial-NoDerivs 4.0 International License (CC BY-NC-ND 4.0), which permits the noncommercial replication and distribution of the article with the strict proviso that no changes or edits are made and the original work is properly cited (including links to both the formal publication through the relevant DOI and the license). See: https://creativecommons.org/licenses/by-nc-nd/4.0/.

\section{References}

1. Garcia-Tsao G, Abraldes JG, Berzigotti A, et al. Portal hypertensive bleeding in cirrhosis: Risk stratification, diagnosis, and management: 2016 practice guidance by the American Association for the study of liver diseases. Hepatology 2017;65:310-35.

2. Bosch J, Garcia-Pagan JC. Prevention of variceal rebleeding. Lancet 2003;361:952-4.

3. de Franchis R, Baveno VI Faculty. Expanding consensus in portal hypertension: Report of the Baveno VI Consensus Workshop: Stratifying risk and individualizing care for portal hypertension. J Hepatol 2015;63:743-52.

4. Tripathi D, Stanley AJ, Hayes PC, et al. U.K. guidelines on the management of variceal haemorrhage in cirrhotic patients. Gut 2015;64:1680-704.

5. Cho H, Nagata N, Shimbo T, et al. Recurrence and prognosis of patients emergently hospitalized for acute esophageal variceal bleeding: A long-term cohort study. Hepatol Res 2016;46:1338-46.

6. Chalasani N, Kahi C, Francois F, et al. Improved patient survival after acute variceal bleeding: a multicenter, cohort study. Am J Gastroenterol 2003;98:653-9.

7. Pfisterer N, Dexheimer C, Fuchs EM, et al. Betablockers do not increase efficacy of band ligation in primary prophylaxis but they improve survival in secondary prophylaxis of 
variceal bleeding. Aliment Pharmacol Ther 2018;47:966-79.

8. Hou MC, Lin HC, Lee FY, et al. Recurrence of esophageal varices following endoscopic treatment and its impact on rebleeding: comparison of sclerotherapy and ligation. J Hepatol 2000;32:202-8.

9. Wang $\mathrm{X}, \mathrm{Wu}$ B. Critical issues in the diagnosis and treatment of liver cirrhosis. Gastroenterol Rep (Oxf) 2019;7:227-30.

10. Branch-Elliman W, Perumalswami P, Factor SH, et al. Rates of recurrent variceal bleeding are low with modern esophageal banding strategies: a retrospective cohort study. Scand J Gastroenterol 2015;50:1059-67.

11. Tajiri T, Yoshida H, Obara K, et al. General rules for recording endoscopic findings of esophagogastric varices (2nd edition). Dig Endosc 2010;22:1-9.

12. Mishra SR, Chander SB, Kumar A, et al. Endoscopic cyanoacrylate injection versus beta-blocker for secondary prophylaxis of gastric variceal bleed: a randomised controlled trial. Gut 2010;59:729-35.

13. Cheng YS, Pan S, Lien GS, et al. Adjuvant sclerotherapy after ligation for the treatment of esophageal varices: a prospective, randomized long-term study. Gastrointest Endosc 2001;53:566-71.

14. Jang WS, Shin HP, Lee JI, et al. Proton pump inhibitor administration delays rebleeding after endoscopic gastric variceal obturation. World J Gastroenterol 2014;20:17127-31.

15. Guo YW, Miao HB, Wen ZF, et al. Procedure-related complications in gastric variceal obturation with tissue glue. World J Gastroenterol 2017;23:7746-55.

16. Tao J, Li J, Chen X, et al. Endoscopic variceal sequential ligation does not increase risk of gastroesophageal reflux disease in cirrhosis patients. Dig Dis Sci 2020;65:329-35.

17. Liu C, Liu Y, Shao R, et al. The predictive value of baseline hepatic venous pressure gradient for variceal rebleeding in cirrhotic patients receiving secondary prevention. Ann Transl Med 2020;8:91.

18. Lv Y, Zuo L, Zhu X, et al. Identifying optimal candidates for early TIPS among patients with cirrhosis and acute variceal bleeding: a multicentre observational study. Gut 2019;68:1297-310.

19. dos Santos JM, Ferreira AR, Fagundes ED, et al. Endoscopic and pharmacological secondary prophylaxis in children and adolescents with esophageal varices. J Pediatr Gastroenterol Nutr 2013;56:93-8.

20. de la Peña J, Brullet E, Sanchez-Hernandez E, et al. Variceal ligation plus nadolol compared with ligation for prophylaxis of variceal rebleeding: a multicenter trial. Hepatology 2005;41:572-8.
21. Park SW, Seo YS, Lee HA, et al. Changes in Cardiac Varices and Their Clinical Significance after Eradication of Esophageal Varices by Band Ligation. Can J Gastroenterol Hepatol 2016;2016:2198163.

22. Xiaoqing Z, Na L, Lili M, et al. Endoscopic Cyanoacrylate Injection with Lauromacrogol for Gastric Varices: LongTerm Outcomes and Predictors in a Retrospective Cohort Study. J Laparoendosc Adv Surg Tech A 2019;29:1135-43.

23. Garcia-Tsao G, Bosch J. Management of varices and variceal hemorrhage in cirrhosis. N Engl J Med 2010;362:823-32. Erratum in: N Engl J Med 2011;364:490.

24. Lo GH. Endoscopic treatments for portal hypertension. Hepatol Int 2018;12:91-101.

25. Kumar A, Jha SK, Sharma P, et al. Addition of propranolol and isosorbide mononitrate to endoscopic variceal ligation does not reduce variceal rebleeding incidence. Gastroenterology 2009;137:892-901.

26. Yoshida H, Mamada $\mathrm{Y}$, Taniai $\mathrm{N}$, et al. A randomized control trial of bi-monthly versus bi-weekly endoscopic variceal ligation of esophageal varices. Am J Gastroenterol 2005;100:2005-9.

27. Lo GH. The role of endoscopy in secondary prophylaxis of esophageal varices. Clin Liver Dis 2010;14:307-23.

28. Hwang JH, Shergill AK, Acosta RD, et al. The role of endoscopy in the management of variceal hemorrhage. Gastrointest Endosc 2014;80:221-7.

29. Kones R, Rumana U, Morales-Salinas A. Confronting the most challenging risk factor: non-adherence. Lancet 2019;393:105-6.

30. Shukla R, Kramer J, Cao Y, et al. Risk and predictors of variceal bleeding in cirrhosis patients receiving primary prophylaxis with non-selective beta-blockers. Am J Gastroenterol 2016;111:1778-87.

31. Polis S, Zang L, Mainali B, et al. Factors associated with medication adherence in patients living with cirrhosis. J Clin Nurs 2016;25:204-12.

32. Zhao C, Jin M, Le RH, et al. Poor adherence to hepatocellular carcinoma surveillance: A systematic review and meta-analysis of a complex issue. Liver Int 2018;38:503-14.

Cite this article as: Wang $\mathrm{X}$, Luo J, Liu C, Liu Y, Wu X, Zheng F, Wen Z, Tian H, Wei X, Guo Y, Li J, Chen X, Tao J, Qi X, Wu B. Impact of variceal eradication on rebleeding and prognosis in cirrhotic patients undergoing secondary prophylaxis. Ann Transl Med 2021;9(7):540. doi: 10.21037/atm20-3401 


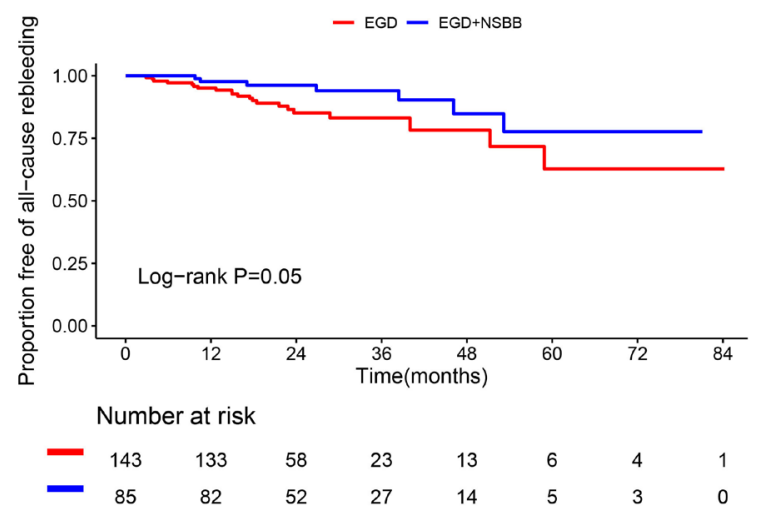

Figure 1 Survival curves of patients showed that the cumulative incidence of all-cause rebleeding was non-significant higher in Endoscopy monotherapy group than in Endoscopy + NSBB group. EGD, esophagogastroduodenoscopy; NSBB, non-selective beta-blocker.

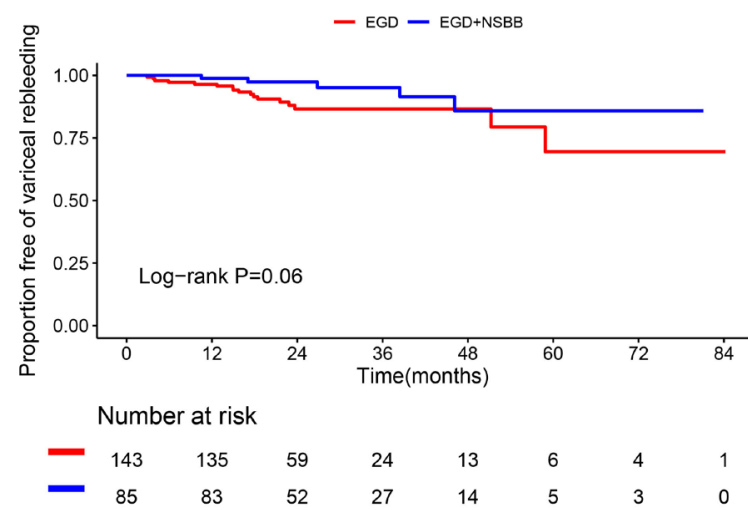

Figure 2 Survival curves of patients showed that the cumulative incidence of variceal rebleeding was non-significant higher in Endoscopy monotherapy group than in Endoscopy + NSBB group. EGD, esophagogastroduodenoscopy; NSBB, non-selective beta-blocker. 
Table S1 Summary of endoscopic sequential therapy

\begin{tabular}{lc}
\hline Characteristics & Total cohort $(\mathrm{n}=228)$ \\
\hline Endoscopic treatment option, $\mathrm{n}(\%)$ & $223(97.8)$ \\
EBL & $91(39.9)$ \\
ECI & $38(16.7)$ \\
EIS & $8.5 \pm 9.5$ \\
Mean time used for variceal eradication (months) & $2.1 \pm 2.0$ \\
Mean interval between sessions (months) & $3.6 \pm 1.8[1-8]$ \\
Sessions of endoscopic therapies (mean, range) & $3.0 \pm 1.5(0-7)$ \\
Sessions of EBL (mean, range) & $18 \pm 8.8(0-43)$ \\
Total number of bands (mean, range) & $0.5 \pm 0.7(0-4)$ \\
Sessions of ECl (mean, range) & $0.3 \pm 0.8(0-6.0)$ \\
Sessions of EIS (mean, range)
\end{tabular}

Data presented as mean \pm SD, range or number of patients (percentage) where appropriate. EBL, Endoscopic banding ligation; ECl, endoscopic cyanoacrylate injection; EIS, endoscopic injection sclerotherapy.

Table S2 Comparison of baseline parameters between fast-VE group and slow-VE group

\begin{tabular}{|c|c|c|c|}
\hline Parameters & Fast-VE group $(\mathrm{n}=128)$ & Slow-VE group $(n=100)$ & $P$ value \\
\hline $\operatorname{PLT}\left(\times 10^{9} / \mathrm{L}\right)$ & $88.7 \pm 46.0$ & $85.0 \pm 39.6$ & 0.522 \\
\hline ALT (U/L) & $40.9 \pm 53.0$ & $37.6 \pm 24.1$ & 0.569 \\
\hline ALB (g/L) & $34.4 \pm 5.3$ & $34.4 \pm 5.5$ & 0.988 \\
\hline MELD score & $11.5 \pm 4.3$ & $10.9 \pm 3.2$ & 0.278 \\
\hline Child-Pugh classification, $\mathrm{n}(\%)$ & & & 0.868 \\
\hline$A$ & $74(57.8)$ & $59(59.0)$ & - \\
\hline $\mathrm{B}$ & $44(34.4)$ & $35(35.0)$ & - \\
\hline
\end{tabular}

Data presented as mean \pm SD or number of patients (percentage) where appropriate. VE, variceal eradication; PLT, platelet; ALT, alanine transaminase; ALB, albumin; TBIL, total bilirubin; MELD, model for end-stage liver disease. 